\title{
Mikrodidaktische Planungen von Lehrenden in der Erwachsenenbildung
}

\section{Theoretische und empirische Annäherungen an ein Forschungsdesiderat}

\section{Tim Stanik (D)}

Eingegangen: 11. August 2016 / Angenommen: 21. November 2016 / Online publiziert: 13. Dezember 2016

(C) Der/die Autor(en) 2016. Dieser Artikel ist eine Open-Access-Publikation.

Zusammenfassung Der Beitrag beschäftigt sich mit einem empirischen Desiderat der Lehr-Lernforschung in der Erwachsenenbildung - dem mikrodidaktischen Planen von Lehrveranstaltungen. Mikrodidaktische Planungen sind komplexe Entscheidungsprozesse, die Einfluss auf das Lehr- und Lernverhalten nehmen. Auf Basis vorliegender Forschungsergebnisse von schulischen Unterrichtsplanungen und Ergebnissen von Befragungen von Lehrenden der Erwachsenenbildung werden Entscheidungsfelder und potentielle Einflussfaktoren auf mikrodidaktische Planungen abgeleitet. Darauf aufbauend werden Forschungsperspektiven und ein Untersuchungsdesign dargelegt, um sowohl die expliziten als auch impliziten Faktoren des Planens von Lehrenden in der Erwachsenenbildung zu analysieren.

Schlüsselwörter Kursplanung · Erwachsenenbildung · Didaktik · Professionalität · Forschung

\section{Planning of courses in adult education}

Approaching a theoretical and empirical desideratum

\begin{abstract}
The article focuses on an empirical desideratum of research on teaching and learning in adult education - the planning of courses. The planning of courses is a complex decision-making process, which influences the teaching and learning behavior. Empirical findings of schoolteachers' planning and results of interviews with trainers in adult education are used to identify elements and potential factors that influenced planning behavior. Based on this, the article develops research
\end{abstract}

T. Stanik $(\bowtie)$

Abteilung Erwachsenenbildung/Weiterbildung, Universität Tübingen,

Münzgasse 11, 72070 Tübingen, Deutschland

E-Mail: tim.stanik@uni-tuebingen.de 
prospects and a research design for analyzing the implicit and explicit factors of planning of courses in adult education.

Keywords Planning courses $\cdot$ Adult education · Didactics $\cdot$ Professionalism · Research

\section{Einleitung}

Mit der Einführung des Diplomstudienganges Pädagogik mit dem Schwerpunkt Erwachsenenbildung stand zunächst das makrodidaktische Programmplanungshandeln im Zentrum der disziplinären Aufmerksamkeit (vgl. Schrader 2010, S. 31). Dies liegt zum einen daran, dass diese Aufgabe von hauptberuflichen Mitarbeitenden übernommen wird, wohingegen die mikrodidaktische Vermittlungstätigkeit von ihren Anfängen bis in die heutige Zeit nebenberuflich konzipiert ist (vgl. Nittel 2000, S. 128). Zum anderen wurde mit dem erwachsenendidaktischen Prinzip der Teilnehmerorientierung und der Durchsetzung des Konstruktivismus als didaktische Leittheorie weniger die Vermittlungstätigkeit als das Arrangieren und Moderieren von Lerngelegenheiten fokussiert. Nach ersten Studien, die sich mit Lehrenden in der Erwachsenenbildung befasst haben (vgl. z. B. Scherer 1987), ist die Kerntätigkeit der Lehre erst in der letzten Dekade zunehmend Gegenstand empirischer Untersuchungen geworden. Diese Untersuchungen betrachten u. a. interaktionsanalytisch Lehr-Lernprozesse (vgl. Kade et al. 2014), rekonstruieren die subjektiven (Wissens-)Theorien der Lehrenden (vgl. Hof 2001), die jeweiligen berufsbiografischen Bedeutungen der Lehrtätigkeit (vgl. Kade 1989) oder fokussieren die organisationskulturellen (vgl. Franz 2016) und die häufig prekären beschäftigungsstrukturellen Rahmenbedingungen der Lehrtätigkeit (vgl. WSF 2005). Weitere Forschungen wenden sich den professionellen Kompetenzen der Lehrenden zu (Kraft et al. 2009) und erarbeiten darauf aufbauend Konzepte, mit denen vorhandene Kompetenzen anerkannt (vgl. Sgier und Lattke 2012) und noch benötigte vermittelt werden sollen (vgl. Schrader et al. 2010).

Ein Forschungsdesiderat sind mikrodidaktische Planungen von Lehrveranstaltungen $^{1}$ in der Erwachsenenbildung, die als Bindeglied zwischen Selbst- bzw. Aufgabenverständnis und professionellem Handeln in den Lehr-Lernprozessen ihre Ausdrucke finden. Während Unterrichtsplanungen von Lehrerinnen und Lehrern fester Bestandteil der schulpädagogischen Theoriebildung und der Lehramtsausbildung sind, wird dieser zentrale Aspekt des professionellen Lehrhandelns in der Erwachsenenbildung eher stiefmütterlich behandelt. Es überwiegen - im Vergleich zur Schulpädagogik in geringer Anzahl - präskriptive Entwürfe bzw. Empfehlungen zur mikrodidaktischen Vorbereitung von Lehr-Lernsituationen. Die vorhandenen

\footnotetext{
1 Wenn im Folgenden von „Lehrveranstaltungsplanungen“ oder den „Planungen von Lehrveranstaltungen“ die Rede ist, sind hiermit sowohl ganze Kurse, Trainings, Schulungen als auch thematisch zusammenhängende Unterrichtseinheiten oder einzelne Kurssitzungen gemeint. Weitere Eingrenzungen sind noch nicht möglich, da empirische Kenntnisse darüber fehlen, wie lang- oder kurzfristig Lehrende in der Erwachsenenbildung ihre Lehrveranstaltungen planen.
} 
Studien über Lehrende in der Erwachsenenbildung ermöglichen Rückschlüsse auf mikrodidaktische Planungen, ohne es jedoch in den Mittelpunkt des Erkenntnisinteresses zu stellen. Lediglich die Studien von Haberzeth (2010) und Hof (2001) nehmen explizit Aspekte der Planung von Lehrveranstaltungen in den Blick. Vor dem Hintergrund, dass insbesondere fachdidaktisches Wissen die Qualität von Unterricht maßgeblich beeinflusst (vgl. Baumert und Kunter 2011), überrascht es, dass mikrodidaktische Planungen sowohl von Lehrerinnen und Lehrern als auch von Erwachsenenbildnerinnen und -bildnern eher vernachlässigte Forschungsfelder sind.

An diesem Forschungsdesiderat setzt der Beitrag an, in dem zunächst mikrodidaktisches Planen definiert und diesbezüglich der empirische Stand sowohl der schul- als auch der erwachsenenpädagogischen Forschung aufgearbeitet wird. Der Diskurs über allgemeine präskriptive didaktische Theoriebildung ist dabei nicht Gegenstand des vorliegenden Beitrags, da nicht normative Begründungen und die Legitimation des geplanten Lehrhandelns behandelt werden, vielmehr sollen empirische Befunde zu mikrodidaktischen Planungen ins Zentrum gerückt werden (vgl. Breidenstein 2008, S. 210). So werden nach einer Synopse der zentralen Ergebnisse und der Ableitung von Forschungsperspektiven methodologische und methodische Überlegungen darüber angestellt, wie didaktische Planungen von Lehrenden in der Erwachsenenbildung analysiert werden können.

\section{Mikrodidaktisches Planen}

Unter mikrodidaktische Planungen lassen sich alle einer intentionalen Lehr-Lernsituation vorausgehenden Maßnahmen subsumieren, die den Vermittlungsakt im Vorfeld strukturieren (vgl. Gassmann 2013, S. 105). Während theoretisch fundierte Unterrichtsplanungen als Garant dafür betrachtet werden, qualitätsvolle Lehr-Lernprozesse zu realisieren (vgl. Peterßen 1982), können diese den Lehrenden auch dazu dienen, die Komplexität des Lehr-Lerngeschehens zu reduzieren, um sich kognitiv und emotional zu entlasten. Darüber hinaus können Lehrplanungen fachliche und methodische Sicherheit bieten und den Lehrveranstaltungen übergreifende Orientierungsrahmen geben (vgl. Seifried 2009, S. 181).

Planungen im Allgemeinen und mikrodidaktische Planungen im Besonderen bewegen sich auf vier analytisch zu unterscheidenden Ebenen:

1. kognitive Orientierungsebene,

2. kognitive Strategieebene,

3. materiale Praxisebene (vgl. Carle 1995, S. 6),

4. soziale Prozessebene.

Mikrodidaktische Planungen sind auf den ersten beiden Ebenen mentale Prozesse der Entscheidungsfindung (vgl. Shavelson und Stern 1981). Es müssen in verschiedenen Feldern Entscheidungen getroffen und diese zueinander in Beziehung gesetzt werden. Zunächst können mikrodidaktische Planungen eine unterschiedliche Reichweite haben, indem sie sich z. B. auf Lehrgänge, auf abgrenzbare Lehr-Lerneinheiten oder auf einzelne Veranstaltungstermine beziehen. Während bei langfristigen Pla- 
nungen eher übergreifende Intentionen, also die Festlegung von Richtzielen im Vordergrund stehen, sind bei kurzfristigen Planungen Feinziele und deren Überprüfung festzulegen. Weitere Entscheidungsfelder betreffen die Auswahl und Aufbereitung der Lerninhalte. Damit sind auch Entscheidungen über zeitliche Strukturierungen, Methoden, Medien und Sozialformen verbunden, die zueinander in Beziehung gesetzt werden müssen (vgl. Nuissl und Siebert 2013, S. 87).

Die materiale Praxisebene umfasst die Erstellung von Lehr- und Lernmaterialien sowie von schriftlichen Planungsentwürfen. Es handelt sich auf diesen Ebenen um einen Prozess, bei dem Erfahrungen, Dispositionen, selbstregulative Fähigkeiten und Professionswissen genutzt werden (vgl. Baumert und Kunter 2006, S. 482). Mikrodidaktisches Planen ist somit auch ein Bereich, in dem fachliche und pädagogische Wissensbestände - ,pedagogical content knowledge“ (Shulman 1986, S. 9 f.) - verknüpft werden müssen.

Neben den kognitiven und materialen Ebenen können mikrodidaktische Planungen auf einer vierten Ebene auch in soziale Prozesse eingebettet sein, wenn beispielsweise Teilnehmende an den Vorbereitungen der Lehrveranstaltung beteiligt werden, die Planungen mit Kolleginnen und Kollegen gemeinsam erfolgen oder mit Auftraggeberinnen und -gebern abgesprochen werden.

Mikrodidaktisches Planen ist zudem in gesellschaftliche und organisationale Kontexte eingebettet (vgl. Schulz 1980, S. 82). Erstere sind in der Erwachsenenbildung an (bildungspolitische) Zielsetzungen (Emanzipation/Bildung, Vermittlung von Faktenwissen, Schlüsselqualifikationen/Kompetenzen) geknüpft (vgl. Nolda 2015, S. 19 ff.), während Letztere die jeweiligen organisationalen Zielsetzungen, Voraussetzungen und Ausstattungen betreffen (vgl. Tietgens 1992, S. 151).

\section{Empirische Befunde über mikrodidaktische Planungen}

Im Folgenden werden zentrale Befunde über schulische Unterrichtsplanungen sowie Ergebnisse aus der Erwachsenenbildungsforschung dargelegt, die explizit und implizit Rückschlüsse auf mikrodidaktisches Planen erlauben.

\subsection{Schulische Unterrichtsplanungen}

Eine erste Metastudie, in der 18 Untersuchungen über schulische Unterrichtsplanungen ausgewertet wurden, zeigte, dass insbesondere die Auswahl der Lerninhalte, der Lernmaterialien und Unterrichtsmethoden im Mittelpunkt steht. Lernziele sind dagegen ein untergeordneter Aspekt und werden, wenn überhaupt, in Interviewstudien mit Lehrerinnen und Lehrern thematisiert (vgl. Shavelson und Stern 1981, S. 479 f.). Außerdem machen weitere Untersuchungen deutlich, dass präskriptive didaktische Modelle häufig nicht bei den Planungen hinzugezogen werden (vgl. Zahorik 1975; John 1991; Tebrügge 2001). Die Zentralität der zu vermittelnden Lerninhalte und die Bedeutung der Methoden bei den Planungen wurden hingegen durch weitere Studien bestätigt (vgl. Bromme 1981; Borko et al. 1990). Im Hinblick auf schülerorientierte Planungsaspekte kommen die Untersuchungen zu unterschiedlichen Befunden. In den Studien von Yinger (1980), Bromme (1981) und Haas (1998) spielen 
diese beispielsweise keine Rolle, während Sardo-Brown (1990) zeigen konnte, dass Interessen und Fähigkeiten von Schülerinnen und Schülern die Planungen von erfahrenen Lehrerinnen und Lehrern durchaus beeinflussen. ${ }^{2}$ Insgesamt konnten bislang aber keine einheitlichen Planungsprozesse von Unterricht identifiziert werden, da diese offensichtlich personenspezifisch und fachabhängig sind (vgl. Stender 2014, S. 84).

Insbesondere die Bedeutung der Berufserfahrungen für Unterrichtsplanungen konnte in einer Reihe von Vergleichsstudien gezeigt werden. So planen Novizen z. B. eher einzelne Unterrichtsstunden als gesamte Unterrichtseinheiten und entwerfen häufiger schriftliche, detaillierte Unterrichtsentwürfe (vgl. Borko und Livingston 1989, S. 475 ff.). Erfahrene Lehrpersonen planen ihren Unterricht hingegen häufiger implizit und schriftliche Unterrichtspläne werden nur für die Verwaltung geschrieben (vgl. Calderhead 1996, S. 713). Auch nutzen erfahrene Lehrpersonen ihre Unterrichtsbeobachtungen häufiger bei den Planungen, wohingegen sich Novizen noch eher an didaktischen Modellen orientieren, was dazu führe, dass kreative Problemlösungen bei den Planungen verloren gingen (vgl. John 2006, S. 495). Novizen greifen auch eher auf die in den Lehrplänen festgelegten Unterrichtsziele zurück. Dies ist insofern ein beachtlicher Befund, als Unterrichtsplanungen als zentraler Link zwischen curricularen Vorgaben und deren Umsetzung in der Unterrichtspraxis betrachtet werden. Kron (2008, S. 300 ff.) unterscheidet diesbezüglich drei Typen von Umgangsweisen mit Lehrplänen: eine strikte Einhaltung, eine Interpretation und einen offenen Umgang.

Am Beispiel der Umsetzung einer Unterrichtsreihe aus dem Lehrplan zur ökonomischen Bildung konnte gezeigt werden, dass ein Drittel der Lehrpersonen den Lehrplan gar nicht bei den Unterrichtsplanungen berücksichtigt, wobei die übrigen zwar die dort hinterlegten Lerninhalte aufgreifen, jedoch von den festgelegten Lernzielen und Lernsituationen abweichen (vgl. Brinkmann-Herz 1984, S. 201). Dass Unterschiede in Unterrichtsplanungen von erfahrenen und unerfahrenen Lehrpersonen nicht auf deren Alter, sondern sich tatsächlich auf ihre Lehrerfahrungen zurückführen lassen, zeigt eine Längsschnittstudie, in der Lehramtsanwärterinnen und -anwärter aus unterschiedlichen Fächern zwei Jahre begleitet worden sind. Die Planungen wurden zunehmend gröber, sodass die Vorbereitung von Unterrichtseinheiten und nicht mehr von -stunden im Vordergrund stand (vgl. Mutton et al. 2011, S. $408 \mathrm{ff}$.).

Neben der Erfahrung legen einige Befunde es auch nahe, dass Unterrichtsfächer Einfluss auf die Unterrichtsplanungen von Lehrerinnen und Lehrern nehmen. In einer Studie, in der Unterrichtsvorbereitungen von Lehramtsstudierenden der Fächer Mathematik und Erdkunde miteinander verglichen wurden, zeigt sich, dass angehende Mathematiklehrerinnen und -lehrer eher eine hierarchische Auffassung von ihrem Fach haben als Lehramtsstudierende des Fachs Erdkunde. So bevorzugen angehende Mathematiklehrerinnen und -lehrer eher instruktive Lehrmethoden bei ihren Planungen, da sie sich an Vorbildern ihrer eigenen Schulkarriere orientieren (vgl. John 1991, S. 314 ff.). Mathematiklehrerinnen und -lehrer orientieren sich bei ihren

\footnotetext{
2 Vor dem Hintergrund der Debatte um Inklusion und Heterogenität bzw. Diversität lässt sich vermuten, dass schülerorientierte Planungsaspekte mittlerweile eine größere Rolle spielen könnten.
} 
Unterrichtsplanungen häufig an einem Standardablauf ihres Unterrichtsfachs (Hausaufgabenbesprechung, Wiederholungen von Themen, Vorstellung neuer Themen im Unterrichtsgespräch, Erläuterungen von Begriffen, Übungsaufgaben, Auswahl von zu stellenden Hausaufgaben). Als zentraler Aspekt der Planung stellt sich dabei die Auswahl von Übungsaufgaben aus Lehrbüchern heraus (vgl. Bromme 1981, S. $190 \mathrm{ff}$.). In einer weiteren Vergleichsstudie wurden Unterrichtsplanungen in den Fächern Mathematik, Chemie und Deutsch miteinander verglichen. Während die zentrale Bedeutung der Auswahl von Übungsaufgaben im Fach Mathematik bei den Planungen bestätigt wird, sind für die Lehrpersonen der beiden anderen Fächer zunächst die logischen Strukturierungen der Stundeninhalte zentral. Außerdem nimmt die Auswahl der Unterrichtsmaterialien für sie einen größeren Stellenwert ein. Überlegungen zur Methodik spielen dagegen in allen drei Fächern eine untergeordnete Rolle (vgl. Tebrügge 2001, S. 205 ff.). Für das Fach Physik konnte auch gezeigt werden, dass die Lehrpersonen häufig damit beginnen, Lernziele festzulegen und daraufhin Experimente planen, wohingegen kaum Entscheidungen über Sozialformen getroffen werden (vgl. Stender 2014, S. 198).

\subsection{Erwachsenenpädagogische Lehrveranstaltungsplanungen}

Wie bereits angesprochen, haben sich bislang explizit nur zwei Studien (vgl. Haberzeth 2010; Hof 2001) mit dem mikrodidaktischen Planen von Lehrenden in der Erwachsenenbildung befasst.

Haberzeth (2010) geht der Frage nach, welche Strategien Lehrende verfolgen, wenn sie Lerninhalte zum Thema ,Lernen“ bei den Planungen in Lehrgegenstände überführen (vgl. ebd., S. 81). Zentrales Ergebnis sind unterschiedliche Thematisierungsstrategien dieser Lerninhalte, die sich in unterschiedlichen Spannungsfeldern bewegen. Während die Spannungsfelder instrumentell vs. reflexiv und individuell vs. strukturell vornehmlich die Auseinandersetzung mit den Lerninhalten betreffen, bestimmen die Strategien sach- vs. methodenbezogen bzw. wissenschafts- vs. erfahrungsbezogen Fragen der didaktischen Aufbereitung (vgl. ebd., S. 228). So sind Fragen des Methoden- und Medieneinsatzes zentrale Entscheidungsfelder, während z. B. die Bedeutung der Inhalte oder deren Wahrheitsgehalt in den Hintergrund treten. Zentral ist außerdem, dass die Inhalte methodisch gut vermittelbar sind, um die Aufmerksamkeit der Teilnehmenden zu erreichen. Im Sample überwiegen außerdem erfahrungsbezogene Strategien, da Lerninhalte auch danach ausgewählt werden, ob sie anschlussfähig für die potenziellen Teilnehmenden sind (vgl. ebd., S. $228 \mathrm{ff}$.). Während hier der zu vermittelnde Lehrgegenstand vorgegeben wurde, konnten Harney und Keiner (1992, S. 217 ff.) bei ehrenamtlichen Kursleitenden in der evangelischen Erwachsenenbildung zeigen, dass „Themenschöpfungen“ sich entweder an Aktualität, an eigener Betroffenheit oder an kirchlichen Autoritäten orientieren. Ausschließliche Orientierungen am eigenen Fachwissen konnten nicht beobachtet werden.

In derselben Studie konnte auch gezeigt werden, dass die Aufnahme einer ehrenamtlichen Lehrtätigkeit (berufs-)biografisch geprägt ist (Jochinke et al. 1992, S. 112 ff.). Biografische Studien über Lehrende bestätigen, dass die Vermittlungstätigkeit, die mit den Veranstaltungen verfolgten Ziele, die stereotypischen Bilder 
von Teilnehmenden und die Art der Vermittlung eng mit den Selbst- und Aufgabenverständnissen als Lehrende verknüpft sind. Ob mit den Veranstaltungen eher die Selbstverantwortlichkeit oder berufliche Handlungsfähigkeit gefördert werden soll und ob Teilnehmende als homogene oder heterogene Lernsubjekte wahrgenommen und behandelt werden, hängt ebenfalls von den jeweiligen Selbstverständnissen ab (vgl. Kade 1989, S. 155 ff.). Dass diese auch konkret die Ebene der Wissensvermittlungen berühren, zeigt die Studie von Bastian (1997), in der vier Typen von Kursleiterinnen und Kursleitern rekonstruiert wurden: die sogenannten „Fachorientierten“, die „FachberaterInnen“, die „Prozessorientierten“ und die „Persönlichkeitsorientierten“. Während die Fachorientierten sich als Sachexperten verstehen, die inhaltliche Lernziele verfolgen und dabei die Voraussetzungen und Interessen der Teilnehmenden nicht berücksichtigen, sind die FachberaterInnen offen gegenüber unterschiedlichen Lerninteressen. Die Prozessorientierten begreifen ihre Kurse als Möglichkeitsraum, in dem die Teilnehmenden sich ausprobieren können, und die Persönlichkeitsorientierten wollen mit ihren Veranstaltungen zur Persönlichkeitsentwicklung beitragen (vgl. ebd., S. 138 ff.).

Dass subjektive Wissenskonzeptionen didaktisches (Planungs-)Handeln beeinflussen, belegt die Studie von Hof (2001). Hier konnten u. a. zwei Kurstypen identifiziert werden, die sogenannten „Fachschulungen“ und die „Persönlichkeitsbildungen“, die sich weder aus den Seminartiteln noch aus den thematisierten Wissensinhalten ablesen lassen (vgl. ebd., S. 103). Stattdessen sind sie davon abhängig, ob die Lehrenden sich als „Fachexperten“ oder als „Methodenexperten“ verstehen. Während erstere es als ihre Aufgabe betrachten, Sachwissen mit Hilfe von lehrzentrierten Unterrichtsmethoden zu vermitteln, wollen die „Methodenexperten“ einen Rahmen schaffen, damit bei den Teilnehmenden Lern- und Bildungsprozesse initiiert werden (vgl. ebd., S. 89). Dabei bedienen sich beide stereotyper Konstruktionen. Von den Methodenexperten werden „Weil-Motive“ und von den Sachexperten „Um-zu-Motive“ bei den Teilnehmenden unterstellt. Inhaltliche didaktische Entscheidungen basieren bei ersteren auf sachbezogenen Überlegungen, um mit Hilfe lehrzentrierter Methoden Wissen zu vermitteln. Bei den unterstellten „Weil-Motiven“ stehen die vermeintlichen Interessen und Probleme der Teilnehmenden im Mittelpunkt der didaktischen Überlegungen. Mit Hilfe entsprechender Methoden sollen hier Möglichkeiten der konkreten Erfahrung in den Lehrveranstaltungen gegeben werden (vgl. ebd., S. 92). Insgesamt zeigt die Studie, dass die Art und Weise wie Lehrveranstaltungen konzipiert und durchgeführt werden, abhängig von den subjektiven Wissenstheorien der Lehrenden und den entsprechenden Unterrichtskonzepten (Training, Unterweisung, Moderation, Beratung) sind (vgl. ebd. S. $129 \mathrm{ff}$.).

Dass didaktisches Handeln auch von den Organisationen abhängig ist, in denen die Lehrveranstaltungen angeboten werden, legen weitere Befunde von Hof (2001) und Haberzeth (2010) nahe. So beeinflussen Veranstaltungsformen, die Bezahlung der Lehrenden und die organisationalen Selbstverständnisse der Weiterbildungseinrichtungen die jeweiligen Unterrichtskonzeptionen (vgl. Hof 2001, S. 111). Dabei sind verschiedene Veranstaltungsformen (Lehrgänge, Blockseminare etc.) eine Besonderheit der Erwachsenenbildung, die prinzipiell inhaltsunabhängig sind, aber Affinitäten zu bestimmten Lernzielen und didaktischen Gestaltungsmöglichkeiten aufweisen (vgl. Tietgens 1992, S. 74). Im Unterschied zur Schule ist das mikrodi- 
daktische Planen von Lehrenden in der Erwachsenenbildung außerdem eine nicht vergütete Tätigkeit, wobei die erheblichen Unterschiede in der Bezahlung der Lehrenden vermuten lassen, dass dies ein weiterer Einflussfaktor ist.

In betrieblich-beruflichen Weiterbildungen nehmen Auftraggeber direkt Einfluss auf die Planungen, da hier die Lehrenden den Nachweis über zielgerichtete Trainings erbringen müssen (vgl. Haberzeth 2010, S. 229). Dass organisationale Selbstverständnisse der Weiterbildungseinrichtungen didaktische Gestaltungen beeinflussen, legen weitere organisationspädagogische Untersuchungen nahe. Je nach ,habituellen Typen“ (Zech 2010, S. 65) unterscheiden sich verschriftlichte organisationale Selbstverständnisse von gelungenen Lernprozessen. Während eine als ,familiär““ charakterisierte Organisation Lernen als selbstbestimmten Prozess versteht, der sich an Wünschen der Teilnehmenden ausrichtet und ohne die Formulierung von Lernzielen auskommt, strebt die als ,dienend“ typisierte Organisation an, die Partizipationsmöglichkeiten ihrer Teilnehmenden zu erhöhen, wohingegen die ,funktionale Organisation“ festgelegte Lernziele möglichst effektiv erreichen will (vgl. Tödt 2010, S. 129 f.).

Weiterbildungsorganisationen nehmen auch implizit als ,konjunktive Erfahrungsräume" Einfluss auf die Lehrveranstaltungen (vgl. Mannheim 1980, S. 223). Eine entsprechende Studie zeigt, dass sich didaktisches Handeln eng an Erwartungen der Teilnehmenden orientieren kann, um diese als Kundinnen und Kunden zu binden. Während hier die Lehrenden ohne Vorgaben der Organisation agieren können, richtet sich didaktisches Handeln in anderen Organisationen stark an Qualitätsentwicklungsprozessen aus. Dort werden mit Bezug auf konstruktivistische Lerntheorien auch Erwartungen an die Didaktik der Lehrenden von den Leitungen kommuniziert. In weiteren Organisationen dominiert dagegen eine prozesshafte Sichtweise auf didaktisches Handeln, das zwar antizipiert und geplant werden kann, aber im Ergebnis offen zu sein hat (vgl. Franz 2013, S. 4 ff.).

\subsection{Synopse: Entscheidungsfelder und Einflussfaktoren mikrodidaktischen Planens}

Fasst man die dargelegten Befunde zusammen, werden die Entscheidungsfelder mikrodidaktischer Planungen (Inhalte, Methoden/Medien, Lernziele, Sozialformen, zeitliche Abfolgen, (Nicht-)Einbezug der Teilnehmenden) durch vier potenzielle Faktoren (Lehrende, Weiterbildungsorganisationen, Fächer/Fachbereiche, Teilnehmende) beeinflusst (vgl. Abb. 1).

Dass Inhalts- und Methodenfragen zentrale Entscheidungsfelder bei schulischen Unterrichtsplanungen sind, unterstützt die beklagte Inhaltslosigkeit der erwachsenendidaktischen Theoriebildung (vgl. Nolda 2001). So ist eines der empirischen gesicherten Erkenntnisse über mikrodidaktische Planungen in der Erwachsenenbildung, dass Lerngegenstände methodische Entscheidungen beeinflussen und in Abhängigkeit zu Antizipationen der Teilnehmenden stehen (vgl. Haberzeth 2010). Inwiefern die Methodenauswahl jedoch zu den anderen Entscheidungsfeldern in Bezug gesetzt wird (vgl. Knoll 2003), ist empirisch nicht beantwortet. Vielmehr zeigen die weiteren Studien über Lehrende in der Erwachsenenbildung, dass Rollenverständnisse (vgl. Bastian 1997) und subjektive Wissenskonzepte (vgl. Hof 2001) sowohl 


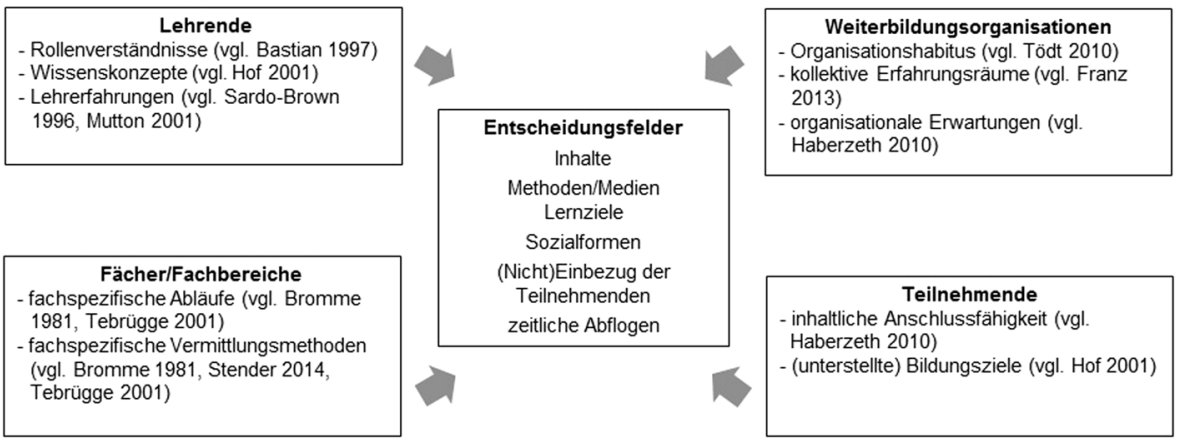

Abb. 1 Entscheidungsfelder und Einflussfaktoren. (Quelle: eigene Darstellung)

die Methodik als auch die Lernziele beeinflussen. Inwiefern sich diese Faktoren bei den Planungen bemerkbar machen und inwiefern die Weiterbildungsorganisationen im- und explizit Einfluss auf die Planungsprozesse nehmen, kann noch nicht gesagt werden. Die vorliegenden Studien (vgl. Franz 2013; Tödt 2010; Haberzeth 2010) lassen diesen Einflussfaktor zumindest als relevante Größe plausibel erscheinen, wenngleich auch dazu dezidierte empirische Überprüfungen notwendig sind. Plausibel erscheint auch, dass (biografisch geprägte) Selbstverständnisse (vgl. Kade 1989) der Lehrenden sich nicht nur auf die Vermittlungstätigkeiten, sondern auch auf die Planungen auswirken.

Die schulpädagogischen Untersuchungen machen deutlich, dass neben dem Unterrichtsfach (vgl. Tebrügge 2001) insbesondere die Lehrerfahrungen sich auf mikrodidaktisches Planen auswirken (vgl. Mutton et al. 2001; Sardo-Brown 1996). Auch dies wäre für die Erwachsenenbildung insofern zu überprüfen, als Lehrende häufig mehrere Jahre hier tätig sind (vgl. WSF 2005, S. 67). Dass sowohl curriculare Vorgaben als auch präskriptive didaktische Modelle kaum bei Unterrichtsplanungen eine Rolle spielen (vgl. John 1991; Tebrügge 2001), ermöglicht eine stärkere vergleichende Perspektive mikrodidaktischen Planens der beiden Bildungssektoren als man es vielleicht zunächst annehmen könnte. Es liegen bislang aber noch keine Erkenntnisse darüber vor, inwiefern nicht doch didaktische Modelle von Lehrenden in der Erwachsenenbildung genutzt werden und wie mit curricularen Vorgaben z. B. in abschlussbezogenen Weiterbildungen umgegangen wird. In diesem Zusammenhang wäre auch zu prüfen, inwiefern sich (nicht) vorhandene pädagogische Ausbildungen auf das Planen auswirken.

Unterschiede zwischen Schule und Erwachsenenbildung sind hinsichtlich des Einbezugs der Lernenden zu erwarten. So spielen schülerzentrierte Planungsaspekte in den meisten Studien eine untergeordnete Rolle (vgl. Bromme 1981, Haas 1998), wohingegen Teilnehmende in der Erwachsenenbildung sowohl bei der Auswahl der Lerninhalte als auch bei der Vermittlungstätigkeit bedacht werden (vgl. Haberzeth 2010).

Wenngleich die dargelegten Erkenntnisse erste Hypothesen bezüglich des mikrodidaktischen Planens in der Erwachsenenbildung nahelegen, bedarf es dennoch explizite, explorative Untersuchungen, um folgende Fragestellungen zu beantworten: 
- Welche Entscheidungsfelder werden bei mikrodidaktischen Planungen (nicht) berücksichtigt?

- Inwiefern werden die Entscheidungsfelder miteinander relationiert?

- Welche Faktoren beeinflussen (nicht) die mikrodidaktischen Planungen?

- In welchen Relationen stehen diese Einflussfaktoren?

Diese forschungsleitenden Fragen zielen damit nicht alleine auf die in der schulpädagogischen Forschung vorherrschende Perspektive auf die kognitiven Planungsprozesse ab, sondern nehmen auch die beeinflussenden Kontextfaktoren in den Blick.

Basierend auf dem Forschungsstand, den skizzierten Desideraten und den daraus abgeleiteten Fragen wurde folgendes Forschungsdesign entwickelt.

\section{Forschungsdesign}

Mikrodidaktische Planungen betrachtet als kognitive, materiale und soziale Prozesse, in denen implizites Erfahrungswissen, deklaratives Professionswissen und wahrscheinlich auch (biografisch geprägte) Auffassungen über fachliche Inhalte, subjektive Rollenverständnisse, Wissenskonzepte als Lehrende, stereotype Konstruktionen der Teilnehmenden sowie Einflüsse der Weiterbildungsorganisationen eine Rolle spielen, sind nicht von außen zu beobachten. Zudem sind mikrodidaktische Planungen - im Gegensatz zur eigentlichen Vermittlungstätigkeit - weder zeitlich noch örtlich eindeutig eingrenzbar (vgl. Gassmann 2013, S. 110).

Aufgrund dieser Bedingungen sowie der noch unzureichenden Studienlage bedarf es eines Untersuchungsdesigns, das es methodologisch ermöglicht, die mikrodidaktischen Planungsprozesse sowohl deskriptiv als auch rekonstruktiv zu analysieren. Deskriptiv können die intentionalen Entscheidungen und die bewussten Relationierungen über Lernziele, Inhalte, Methoden etc. sowie die dabei reflexiv berücksichtigten Einflussfaktoren erfasst werden. Zusätzlich sind aber rekonstruktive Verfahren notwendig, um die latenten Einflüsse der weiteren Einflussfaktoren auf die Planungen zu rekonstruieren. Shavelson und Stern (1981, S. 44 f.) weisen darauf hin, dass nur weil Lehrerinnen und Lehrer in Darstellungen ihrer Unterrichtsplanungen nicht explizit über Lernziele sprechen, dies noch lange nicht bedeute, dass diese nicht doch die Planungen beeinflussen. So scheinen Lernziele bei Unterrichtsplanungen eng an Inhalte gebunden zu sein bzw. häufig mit ihnen gleichgesetzt zu werden, im Sinne von „stoffgekoppelten Zielen“ (Haas 2005, S. 8). Auch ist davon auszugehen, dass die Umsetzungen der Planungen und deren kritische Reflexionen dazu führen, dass erfolgreiche Lehrkonzeptionen sukzessive in Handlungsskripte überführt werden, die die bewussten Planungen und Entscheidungen ablösen und daher nicht mehr vollständig reflexiv verfügbar sind (vgl. Stender 2014, S. 65).

Damit stellt sich auch die Frage nach geeigneten Methoden zur Erfassung von mikrodidaktischen Planungen. Unterrichtsplanungen von Lehrerinnen und Lehrern werden häufig mit der Methode des lauten Denkens untersucht (vgl. z. B. Bromme 1981; Haas 1998; Tebrügge 2001), die zuweilen durch (stimulated recall) Interviews oder durch Fragebogenerhebungen ergänzt werden. Hierbei ist methodologisch noch strittig, inwiefern hinreichende Artikulationsmöglichkeiten bei allen Lehrenden zur 
Verfügung stehen, um diese komplexen (kognitiven) Prozesse zu versprachlichen. Auch wird noch kontrovers diskutiert, inwiefern sich Verbalisierungen auf die Planungsprozesse auswirken (vgl. Konrad 2010, S. 486). Aufgrund dieser methodologisch ungelösten Probleme, stellt das Problemzentrierte Interview als theoriegenerierendes Verfahren mit seinen spezifischen Merkmalen einen alternativen Ansatz zur Erhebung mikrodidaktischen Planens dar. Mithilfe dieser leitfadengestützten Interviewmethode wird es möglich, Aussagen über Orientierungsmuster und Planungspraxen der Lehrenden zu erhalten und diese bereits in der Interviewsituation mit dem theoretischen Vorwissen um Lehrveranstaltungsplanungen durch Nachfragen in Bezug zu setzen (vgl. Witzel 2000). Neben den Interviews soll auch auf schriftliche Planungsentwürfe - insofern diese erstellt werden - datentriangulativ zurückgegriffen werden, um damit auch die materiale Praxisebene zu berücksichtigen.

Eine der zentralen Herausforderungen ist, aufgrund der fachlich-inhaltlichen Vielfältigkeit der Lehrveranstaltungen und der Heterogenität der Lehrenden in der Erwachsenenbildung, die Zusammenstellung eines aussagekräftigen Untersuchungssamples. Dieser soll damit begegnet werden, dass ein Sample von Lehrenden nicht fachbereichsbezogen, sondern mit Hilfe von wissenstypologischen Merkmalen der Lehrveranstaltungen (Handlungswissen, Interaktionswissen, Identitätswissen, Orientierungswissen) gebildet wird (vgl. Schrader 2003), um eine ,didaktisch akzentuierte Analyse“ (Schrader und Berzbach 2005, S. 6) zu ermöglichen. Aufgrund der dargelegten Befunde müssen im Sample Lehrende berücksichtigt werden, die für unterschiedliche Weiterbildungsorganisationen tätig werden, unterschiedliche pädagogische Qualifikationen und Lehrerfahrungen haben.

Wie bereits erwähnt, muss die Auswertung der gewonnenen Daten methodentriangulativ erfolgen, indem mit Hilfe einer strukturierenden Inhaltsanalyse die genannten Entscheidungsfelder und Einflussfaktoren kategorisiert und zueinander in Beziehung gesetzt werden (vgl. Kuckartz 2012). Für die Aufschlüsselung der impliziten Planungsaspekte und Einflussfaktoren bedarf es rekonstruktiver Analyseverfahren, wie der dokumentarischen Methode, mit der auch die latenten „Orientierungsrahmen“ (Bohnsack 2003, S. 135) der Planungsprozesse rekonstruierbar sind.

Ziel dieses explorativen, triangulativen Forschungsdesigns ist zunächst die Entwicklung einer „materialen Theorie“ (Glaser und Strauss 1998, S. 42) mikrodidaktischen Planens in der Erwachsenenbildung. Diese ist dann vor dem Hintergrund des Diskurses der allgemeinen didaktischen Theoriebildungen zu diskutieren und in anschließenden quantifizierenden Erhebungen zu verifizieren.

Open Access Dieser Artikel wird unter der Creative Commons Namensnennung 4.0 International Lizenz (http://creativecommons.org/licenses/by/4.0/deed.de) veröffentlicht, welche die Nutzung, Vervielfältigung, Bearbeitung, Verbreitung und Wiedergabe in jeglichem Medium und Format erlaubt, sofern Sie den/die ursprünglichen Autor(en) und die Quelle ordnungsgemäß nennen, einen Link zur Creative Commons Lizenz beifügen und angeben, ob Änderungen vorgenommen wurden. 


\section{Literatur}

Bastian, H. (1997). Kursleiterprofile und Angebotsqualität. Bad Heilbrunn: Klinkhardt.

Baumert, J., \& Kunter, M. (2006). Stichwort: Professionelle Kompetenz von Lehrkräften. Zeitschrift für Erziehungswissenschaft, 9(4), 469-520.

Baumert, J., \& Kunter, M. (2011). Das mathematikspezifische Wissen von Lehrkräften, kognitive Aktivierung im Unterricht und Lernfortschritte von Schülerinnen und Schülern. In M. Kunter, J. Baumert, W. Blum \& M. Neubrand (Hrsg.), Professionelle Kompetenz von Lehrkräften. Ergebnisse des Forschungsprogramms COACTIV (S. 163-192). Münster: Waxmann.

Bohnsack, R. (2003). Rekonstruktive Sozialforschung. Einführung in Methodologie und Praxis qualitativer Forschung. Opladen: utb.

Borko, H., \& Livingston, C. (1989). Cognition and Improvisation: Differences in Mathematics Instruction by Expert and Novice Teachers. American Educational Research Journal, 26(4), 473-498.

Borko, H., Livingston, C., \& Shavelson, R. J. (1990). Teachers' Thinking About Instruction. Remedial and Special Education, 11(6), 40-49.

Breidenstein, G. (2008). Allgemeine Didaktik und praxeologische Unterrichtsforschung. In M. A. Meyer, M. Prenzel \& S. Hellekamps (Hrsg.), Perspektiven der Didaktik (S. 201-215). Wiesbaden: Springer.

Brinkmann-Herz, D. (1984). Der Einfluss innovativer Lehrpläne auf die Unterrichtsplanung der Lehrer. Eine entscheidungstheoretische Untersuchung am Beispiel eines Lehrplanes zur ökonomischen Bildung in der Hauptschule. Frankfurt: Peter Lang.

Bromme, R. (1981). Das Denken von Lehrern bei der Unterrichtsvorbereitung: Eine empirische Untersuchung zu kognitiven Prozessen von Mathematiklehrern. Weinheim: Beltz.

Calderhead, J. (1996). Teachers' beliefs and knowledge. In D. Berliner \& R. C. Calfee (Hrsg.), Handbook of educational psychology (S. 709-725). New York: Simon and Schuster.

Carle, U. (1995). „Mein Lehrplan sind die Kinder“ - Eine Analyse der Planungstätigkeit von Lehrerinnen und Lehrern an Förderschulen. Weinheim: Deutscher Studienverlag.

Franz, J. (2013). Didaktisches Handeln in Organisationen Allgemeiner Erwachsenenbildung. Erste Ergebnisse. Magazin erwachsenenbildung.at., 20, 1-12. http://erwachsenenbildung.at/magazin/13-20/07_ franz.pdf.

Franz, J. (2016). Kulturen des Lehrens. Eine Studie zu kollektiven Lehrorientierungen in Organisationen Allgemeiner Erwachsenenbildung. Bielefeld: W. Bertelsmann.

Gassmann, C. (2013). Erlebte Aufgabenschwierigkeit bei der Unterrichtsplanung. Eine qualitativ-inhaltsanalytische Studie zu den Praktikumsphasen der universitären Lehrerbildung. Wiesbaden: Springer.

Glaser, B.G., \& Strauss, A. L. (1998). Grounded Theory: Strategien qualitativer Forschung. Bern: Huber.

Haas, A. (1998). Unterrichtsplanung im Alltag: Eine empirische Untersuchung zum Planungshandeln von Hauptschul-, Realschul- und Gymnasiallehrern. Regensburg: Roderer.

Haas, A. (2005). Unterrichtsplanung im Alltag von Lehrerinnen und Lehrern. In A. A. Huber (Hrsg.), Vom Wissen zum Handeln - Ansätze zur Überwindung der Theorie-Praxis-Kluft in Schule und Erwachsenenbildung (S. 5-19). Tübingen: Huber.

Haberzeth, E. (2010). Thematisierungsstrategien im Vermittlungsprozess. Empirische Analysen zum Umgang mit Wissen im Planungsprozess von Weiterbildungsangeboten. Baltmannsweiler: Schneider Verlag Hohengehren.

Harney, K., \& Keiner, E. (1992). Zum Profil nicht-hauptberuflicher Arbeit in der kirchlichen Erwachsenenbildung. In D. H. Jütting (Hrsg.), Situation, Selbstverständnis, Qualifizierungsbedarf. Nicht-hauptberufliche MitarbeiterInnen in der Deutschen Evangelischen Arbeitsgemeinschaft für Erwachsenenbildung Empirische Studien. (S. 197-227). Frankfurt: Peter Lang.

Hof, C. (2001). Konzepte des Wissens: eine empirische Studie zu den wissenstheoretischen Grundlagen des Unterrichts. Bielefeld: W. Bertelsmann.

Jochinke, M., Jütting, D.H., \& Knoblauch-Flach, A. (1992). Die Untersuchung der nicht-hauptberuflichen MitarbeiterInnen in der Erwachsenenbildung der DEAE. In D. H. Jütting (Hrsg.), Situation, Selbstverständnis, Qualifizierungsbedarf. Nicht-hauptberufliche MitarbeiterInnen in der Deutschen Evangelischen Arbeitsgemeinschaft für Erwachsenenbildung - Empirische Studien. (S. 3-196). Frankfurt a.M.: Peter Lang.

John, P. D. (1991). A qualitative study of British student teachers' lesson planning perspectives. Journal of Education for Teaching, 17(3), 301-320.

John, P. D. (2006). Lesson planning and the student teacher: re-thinking the dominant model. Journal of Curriculum Studies, 38(4), 483-498.

Kade, J. (1989). Kursleiter und die Bildung Erwachsener: Fallstudien zur biographischen Bedeutung der Erwachsenenbildung. Bad Heilbrunn: Klinkhardt. 
Kade, J., Nolda, S., Dinkelaker, J., \& Herrle, M. (Hrsg.). (2014). Videographische Kursforschung. Empirie des Lehrens und Lernens Erwachsener. Stuttgart: Kohlhammer.

Knoll, J. (2003). Kurs- und Seminarmethoden. Ein Trainingsbuch zur Gestaltung von Kursen und Seminaren, Arbeits- und Gesprächskreisen (10. Aufl.). Weinheim: Beltz.

Konrad, K. (2010). Lautes Denken. In G. Mey \& K. Mruck (Hrsg.), Handbuch Qualitative Forschung in der Psychologie (S. 476-490). Wiesbaden: Springer.

Kraft, S., Seitter, W., \& Kollewe, L. (2009). Professionalitätsentwicklung des Weiterbildungspersonals. Bielefeld: W. Bertelsmann.

Kron, F. W. (2008). Grundwissen Didaktik. Bd. 5. München: Reinhard.

Kuckartz, U. (2012). Qualitative Inhaltsanalyse. Methoden, Praxis, Computerunterstützung. Weinheim: Beltz.

Mannheim, K. (1980). Strukturen des Denkens. Frankfurt: Suhrkamp.

Mutton, T., Hagger, H., \& Burn, K. (2011). Learning to plan, planning to learn: the developing expertise of beginning teachers. Teachers and Teaching, 17(4), 399-416.

Nittel, D. (2000). Von der Mission zur Profession? Stand und Perspektiven der Verberuflichung in der Erwachsenenbildung. Bielefeld: W. Bertelsmann.

Nolda, S. (2001). Vom Verschwinden des Wissens in der Erwachsenenbildung. Zeitschrift für Pädagogik, 47(1), 101-120.

Nolda, S. (2015). Einführung in die Theorie der Erwachsenenbildung (3. Aufl.). Darmstadt: WBG.

Nuissl, E., \& Siebert, H. (2013). Lehren an der VHS. Bielefeld: W. Bertelsmann.

Peterßen, W.H. (1982). Handbuch der Unterrichtsplanung. Grundfragen, Modell, Stufen, Dimensionen (6. Aufl.). München: Ehrenwirth.

Sardo-Brown, D. (1990). Experienced teachers' planning practices: A US survey. Journal of Education for Teaching, 16(1), 57-71.

Sardo-Brown, D. (1996). A longitudinal study of novice secondary teachers' planning: year two. Teaching and Teacher Education, 12(5), 519-530.

Scherer, A. (1987). Freie Mitarbeiter in der Erwachsenenbildung. Frankfurt a.M.: Peter Lang.

Schrader, J. (2003). Wissensformen in der Weiterbildung. W. Gieseke (Hrsg.), Institutionelle Innensichten der Weiterbildung (S. 228-253). Bielefeld: W. Bertelsmann.

Schrader, J. (2010). Fortbildung von Lehrenden der Erwachsenenbildung: Notwendig? Sinnvoll? Möglich? In J. Schrader, R. Hohmann \& S. Hartz (Hrsg.), Mediengestützte Fallarbeit: Konzepte, Erfahrungen und Befunde zur Kompetenzentwicklung von Erwachsenenbildnern (S. 25-68). Bielefeld: W. Bertelsmann.

Schrader, J., \& Berzbach, F. (2005). Empirische Lernforschung in der Erwachsenenbildung. Bonn. http:// www.die-bonn.de/esprid/dokumente/doc-2005/schrader05_01.pdf. Zugegriffen: 12.12.2016.

Schrader, J., Hohmann, R., \& Hartz, S. (Hrsg.). (2010). Mediengestützte Fallarbeit: Konzepte, Erfahrungen und Befunde zur Kompetenzentwicklung von Erwachsenenbildnern. Bielefeld: W. Bertelsmann.

Schulz, W. (1980). Unterrichtsplanung. München: Urban und Schwarzenberg.

Seifried, J. (2009). Unterrichtsplanung von (angehenden) Lehrkräften an kaufmännischen Schulen. Zeitschrift für Berufs- und Wirtschaftspädagogik, 105(2), 179-197.

Sgier, I., \& Lattke, S. (Hrsg.). (2012). Professionalisierungsstrategien der Erwachsenenbildung in Europa. Entwicklungen und Ergebnisse aus Forschungsprojekten. Bielefeld: W. Bertelsmann.

Shavelson, R. J., \& Stern, P. (1981). Research on teachers' pedagogical thoughts, judgments, decisions, and behavior. Review of Educational Research, 51(4), 455-498.

Shulman, L.S. (1986). Those who understand: Knowledge growth in teaching. Educational Researcher, 15(2), 4-14.

Stender, A. (2014). Unterrichtsplanung: Vom Wissen zum Handeln - Theoretische Entwicklung und empirische Überprüfung des Transformationsmodells der Unterrichtsplanung. Berlin: Logos.

Tebrügge, A. (2001). Unterrichtsplanung zwischen didaktischen Ansprüchen und alltäglicher Berufsanforderung: Eine empirische Studie zum Planungshandeln von Lehrerinnen und Lehrern in den Fächern Deutsch, Mathematik und Chemie. Frankfurt a.M.: Peter Lang.

Tietgens, H. (1992). Reflexionen zur Erwachsenendidaktik. Bad Heilbrunn: Klinkhardt.

Tödt, K. (2010). Damit Lernen gelingt: Wie beschreiben Weiterbildungsorganisationen ihre Lehr-Lernprozesse? In R. Zech, C. Dehn, K. Tödt, S. Rädiker, M. Mrugalla \& J. Schunter (Hrsg.), Organisationen in der Weiterbildung. Selbstbeschreibungen und Fremdbeschreibungen (S. 102-131). Wiesbaden: Springer.

Wirtschafts- und Sozialforschung (WSF) (2005). Erhebung zur beruflichen und sozialen Lage von Lehrenden in den Weiterbildungseinrichtungen. Schlussbericht. https://www.phil-fak.uni-duesseldorf.de/ fileadmin/Redaktion/Institute/Sozialwissenschaften/BF/Lehre/Materialien/Weiterbildung/berufliche_ und_soziale_lage_von_lehrenden_in_weiterbildungseinrichtungen.pdf. Zugegriffen: 12.12.2016. 
Witzel, A. (2000). Das problemzentrierte Interview. Forum Qualitative Sozialforschung/Forum: Qualitative Social Research, 1(1), Art. 22. http://www.qualitative-research.net/fqs-texte/1-00/1-00witzel-d. pdf.

Yinger, R. J. (1980). A study of teacher planning. The Elementary School, 80(3), 107-127.

Zahorik, J. A. (1975). Teachers' planning models. Educational Leadership, 33(2), 134-139.

Zech, R. (2010). Organisationen in der Weiterbildung. Selbstbeschreibungen und Fremdbeschreibungen. In R. Zech, C. Dehn, K. Tödt, S. Rädiker, M. Mrugalla \& J. Schunter (Hrsg.), Organisationen in der Weiterbildung. Selbstbeschreibungen und Fremdbeschreibungen (S. 11-71). Wiesbaden: Springer. 